\title{
Toward Optimal Mobility-Aware VM Placement and Routing in Space-Air-Ground Integrated Networks
}

\author{
Amir Varasteh*, Sandra Hofmann ${ }^{\dagger}$, Nemanja Deric*$^{*}$, Andreas Blenk*, Dominic Schupke ${ }^{\dagger}$ \\ Wolfgang Kellerer*, and Carmen Mas Machuca* \\ ${ }^{*}$ Chair of Communication Networks, Technical University of Munich, Germany \\ Email: \{amir.varasteh, nemanja.deric, andreas.blenk, wolfgang.kellerer, cmas\}@tum.de \\ ${ }^{\dagger}$ Airbus, Munich, Germany \\ Email: \{sandra.s.hofmann, dominic.schupke\}@airbus.com
}

\begin{abstract}
Today's airplane passengers are also demanding Internet-based services such as video streaming or web services. While providing cost-efficient solutions in core networks is already quite challenging, providing Internet-based services in the air reaches even a new dimension of problem complexity. Not only routing and placement problems need to be solved in the core network, but also new types of link connections, i.e., AirTo-Ground (A2G) connections from satellites and Direct Air-ToGround (DA2G) links need to be considered while provisioning and managing Internet-based services. Moreover, in such SpaceAir-Ground Integrated Networks (SAGIN), the airplanes as service endpoints are changing their locations in the network over time. Hence, to always provide cost-efficient and reliable resource management solutions, routing and service placement problems, i.e., placement of Virtual Machines (VMs), need to be constantly solved over time. In this work, we demonstrate the trade-offs among solutions of different optimization algorithms, which jointly determine routing, service placement, and service migrations for flying airplanes in a Space-Air-Ground Integrated Network. Furthermore, we demonstrate how service networks dynamically adapt according to moving airplanes.
\end{abstract}

Index Terms-space-air-ground, mobility, multi-period optimization, migration, placement

\section{INTRODUCTION AND PROBLEM DESCRIPTION}

Internet-based services, such as video streaming and web browsing, are offered by more and more airlines today. Additionally, there are operational services that need to communicate to the ground (e.g., monitoring data for flight status). All these services are hosted by Virtual Machine (VM) instances located in datacenters (DCs), which are distributed in the ground network (See Fig. 1). Moreover, each type of service has different Quality of Service (QoS) requirements (e.g., delay), which have to be guaranteed.

Fig. 1 depicts a Space-Air-Ground Integrated Network (SAGIN) [1] where an airplane is flying over the ground network. The airplane has two Air-To-Ground (A2G) link alternatives in order to communicate to VMs running in DCs on the ground: $i$ ) Satellite links (e.g., Low Earth Orbit (LEO)) and ii) Direct AirTo-Ground (DA2G) links. Satellite links can provide network bandwidth globally to the flying airplanes. The satellites relay the received traffic from the airplanes toward their gateways, also located in the ground core network (See Fig. 1). As a second alternative, a DA2G node can establish a direct connection to the airplane, whenever the distance allows the communication. The DA2G connection has higher link capacity and lower delay and less cost than satellite connections [2].

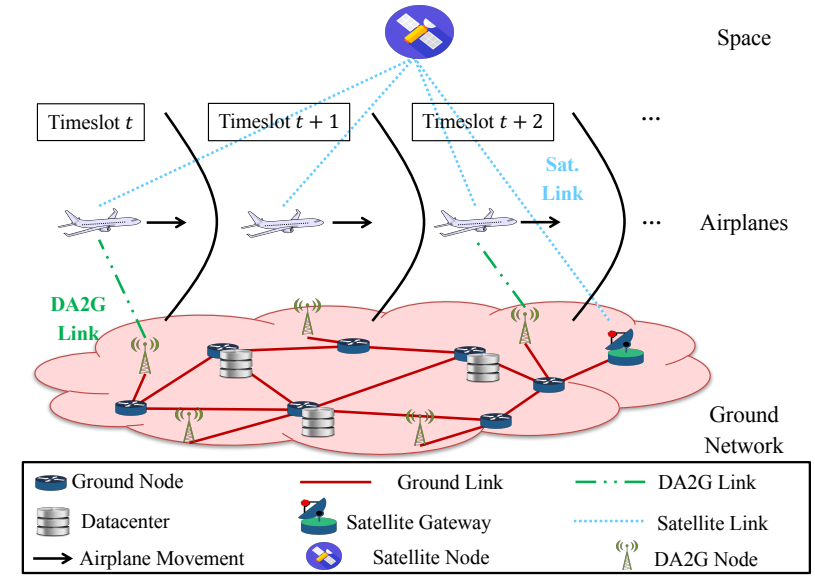

Fig. 1: The Considered Space-Air-Ground Integrated Network.

However, in case of overloaded DA2G connections, satellite connections may have to be used as alternatives still. In such a scenario, the placement of the VMs on DCs should not only consider the available DC resources and their costs, but also the routing to the satellite gateway, in order to guarantee the QoS requirements. In order to not interrupt services, interDC VM migrations can adapt the VM placements and the routing based on the mobility patterns and the future positions of airplanes. Since VM migrations might be costly in terms of network bandwidth, energy consumption, etc. [3], also the cost of VM migrations should be taken into account.

Therefore, by considering the mobility of airplanes (different locations at different time-slots), and the heterogeneity of the SAGIN architecture, four questions should be addressed: i) How many VMs of each service type have to be placed on each DC for each time-slot? ii) Considering bandwidth and delay requirements, which path should be used for airplaneto-VM communications? iii) Is a VM migration necessary? If yes, from which DC to which? iv) How to jointly reply to the aforementioned questions in a cost-efficient manner?

In our previous work [4], we proposed a mathematical formulation to determine the optimal VM placement, routing, and required VM migration(s) at each time-slot $t \in \mathcal{T}$, where $\mathcal{T}$ is the set of time-slots (i.e., duration) of a flight (see Fig. 1). Based on the known future positions of airplanes, we formulated the Mobility-Aware Joint Service Placement and Routing (MA-JSPR) approach as an Integer Linear Program (ILP) with the objective of minimizing the total incurred 


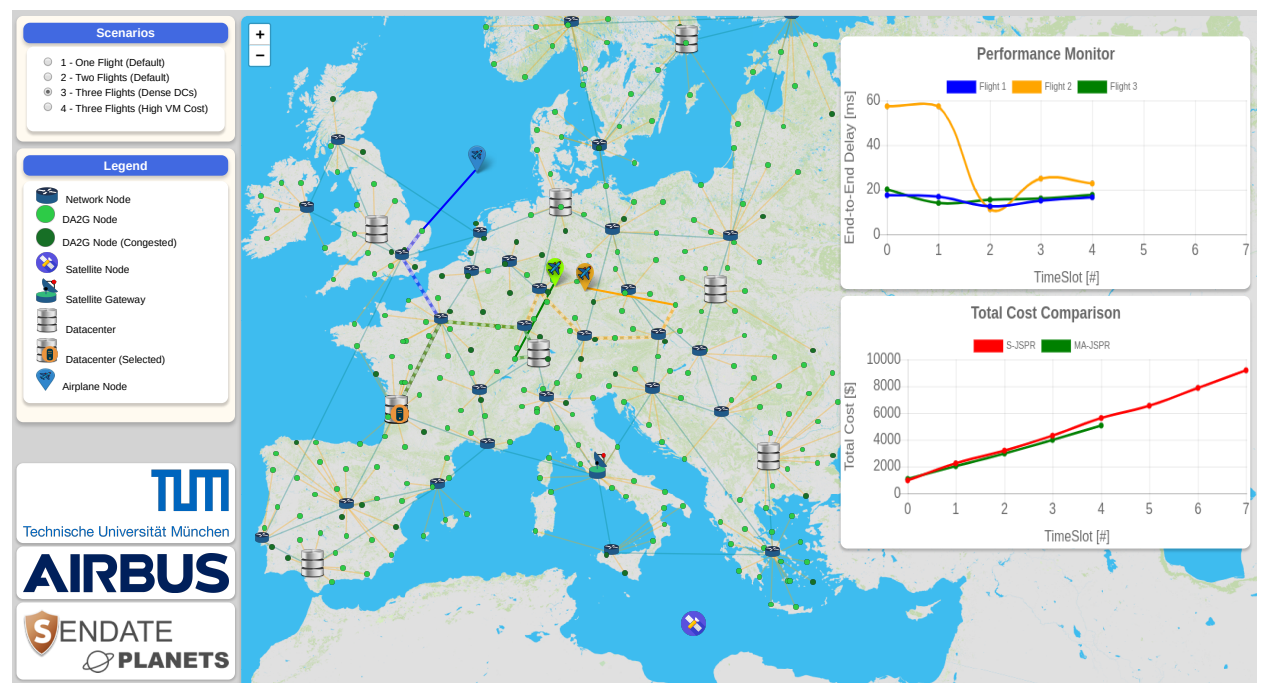

Fig. 2: A Screenshot of the Proposed Demonstrator.

long-term cost. We considered the following costs: end-to-end routing cost from the airplane to the $\mathrm{DC}, \mathrm{VM}$ deployment cost, and VM migration cost between DCs when required.

In this demo, we present the Graphical User Interface (GUI) of our proposed MA-JSPR. This GUI provides a simple and clear way to demonstrate the optimal routing, VM placement, and migration decisions during the airplane(s) flight, in a realistic SAGIN for different scenarios.

\section{THE DEMONSTRATOR}

Fig. 2 shows a screenshot of the GUI ${ }^{1}$. The GUI can be grouped into three parts: a realistic European-based SAGIN, a scenario selection part, and the output part.

A. Realistic European-based SAGIN: The central part depicts a European-based SAGIN map, which contains several components: a European fixed network interconnecting ground core nodes (Cost266 [5]), some of them containing DCs [6], DA2G nodes distributed over Europe [7], a representative satellite node and its gateway in Rome, Italy [8]. In this SAGIN, while three airplanes are flying over Europe, the optimal routing, VM placement and migration decisions at each $t \in \mathcal{T}$ are depicted on the map.

B. Scenarios: The upper left side lists three exemplary scenarios differing in three aspects: $i$ ) Number of flights over $\mathcal{T}, i i)$ number and location of DCs, and iii) cost values, in particular, VM deployment, and migration costs.

C. Output: While a number of airplanes (three in Fig. 2) are flying over Europe, the optimal (pre-computed) solution for routing, VM placement and migration decisions are visualized on the GUI. It can be seen how these decisions are adapted over time, with the minimum incurred cost. Moreover, a delay monitor (upper chart) shows the end-to-end delay always below the threshold ( $80 \mathrm{~ms}$ in this case) for all flights. The lower chart shows the cost comparison of MA-JSPR with respect to the solution of the State Joint Service Placement and Routing algorithm (S-JSPR). S-JSPR solves the problem

\footnotetext{
${ }^{1}$ Video of the Demo: https://wiki.lkn.ei.tum.de/public:sagin_demo:start
}

at each $t$ without considering the whole $\mathcal{T}$. It can be seen that MA-JSPR can reduce the total cost.

The Presentation: We demonstrate the functionality of the proposed framework to jointly determine routing, VM placement and migration for one or three airplanes over a timehorizon $\mathcal{T}=3.5$ hour, $\Delta t=0.5$ hour. As different particular scenarios, we focus on varying input costs of VM deployment and migrations and compare the achieved end-to-end delay and total costs. We also show how the number of DCs can affect the output of the framework. The source code of the presented framework is publicly available.

We believe this demonstration can help airline companies to try out and evaluate different network resource management strategies for their network services. Moreover, the approach can also be applied to other scenarios where networks change dynamically, such as trains, ships, or self-driving cars.

\section{ACKNOWLEDGMENT}

This work was supported in part under the Celtic-Plus subproject SEcure Networking for a DATacenter cloud in Europe (SENDATE)-PLANETS (Project ID 16KIS0261/16KIS0461) funded by the German Federal Ministry of Education and Research (BMBF). The authors alone are responsible for the content of the paper.

\section{REFERENCES}

[1] J. Liu et. al. Space-Air-Ground Integrated Network: A Survey. IEEE Communications Surveys and Tutorials, 2018

[2] E. Dinc et. al. Multi-user Beamforming and Ground Station Deployment for 5G Direct Air-to-Ground Communication. In Global Communications, IEEE International Conference on, pages 1-7, 2017.

[3] A. Varasteh et. al. Server consolidation techniques in virtualized data centers: A survey. IEEE Systems Journal, 11(2):772-783, 2017.

[4] A. Varasteh et. al. Mobility-Aware Joint Service Placement and Routing in Space-Air-Ground Integrated Networks. In International Conference on Communications (ICC). IEEE, 2019.

[5] SNDLib Topologies, Available: https://sndlib.zib.de.

[6] Datacenter Map, Available: https://www.datacentermap.com/cloud.html.

[7] U. Mansmann. Fast Internet for aircraft in Europe, Available: https://goo.gl/KUFGGL, 2018.

[8] The Dream of Affordable Internet Access for Everyone is Getting Closer, Available: https://goo.gl/eTkRnL, 2017. 